\title{
On Mixed Joint Discrete Universality for a Class of Zeta-Functions: a Further Generalization
}

\author{
Roma Kačinskait: $\dot{\mathrm{e}}^{a}$ and Kohji Matsumoto ${ }^{b}$ \\ ${ }^{a}$ Vytautas Magnus University \\ Vileikos g. 8, LT-44404 Kaunas, Lithuania \\ ${ }^{b}$ Nagoya University \\ Furocho, Chikusa-ku, 464-8602 Nagoya, Japan \\ E-mail(corresp.): kohjimat@math.nagoya-u.ac.jp \\ E-mail: roma.kacinskaite@vdu.lt
}

Received December 16, 2019; revised July 28, 2020; accepted July 30, 2020

\begin{abstract}
We present the most general at this moment results on the discrete mixed joint value-distribution (Theorems 5 and 6) and the universality property (Theorems 3 and 4) for the class of Matsumoto zeta-functions and periodic Hurwitz zetafunctions under certain linear independence condition on the relevant parameters, such as common differences of arithmetic progressions, prime numbers etc.
\end{abstract}

Keywords: discrete shift, Matsumoto zeta-function, periodic Hurwitz zeta-function, simultaneous approximation, Steuding class, value distribution, weak convergence, universality.

AMS Subject Classification: 11M06; 11M41; 11M35; 41A30; 30E10.

\section{Introduction}

In analytic number theory, one of the most interesting and popular subjects is the so called universality property of various zeta- and $L$-functions in Voronin's sense (see [27]). In particular the mixed joint universality is a rather hot topic today. Recall that the first results in this direction were obtained by J. Sander and J. Steuding (see [25]) and independently by H. Mishou (see [24]). They proved that the pair of analytic functions is simultaneously approximable by the shifts of the Riemann zeta-function $\zeta(s)$ and the Hurwitz zeta-function $\zeta(s, \alpha)$ with certain real $\alpha$ (as it is well-known the function $\zeta(s)$ has the Euler type product expansion over primes, while the function $\zeta(s, \alpha)$ in general does not; from this facts the term "mixed joint" arises). 
In the series of previous works by the authors (see $[9,10,11,12])$, the tuple consisting from the wide class of the Matsumoto zeta-functions $\varphi(s)$ and the periodic Hurwitz zeta-functions $\zeta(s, \alpha ; \mathfrak{B})$ is considered in the frame of the studies on the value distribution of such pair, as well as the universality property and the functional independence.

Let $s=\sigma+i t$ be a complex variable and by $\mathbb{P}, \mathbb{N}, \mathbb{Q}, \mathbb{R}$ and $\mathbb{C}$ denote the set of all prime numbers, positive integers, rational numbers, real numbers and complex numbers, respectively. Suppose that $\alpha, 0<\alpha \leq 1$, is a fixed real number. Recall the definitions of both the functions under our interest - the Matsumoto zeta-functions $\varphi(s)$ and the periodic Hurwitz zeta-functions $\zeta(s, \alpha ; \mathfrak{B})$.

For $m \in \mathbb{N}$, let $g(m) \in \mathbb{N}, f(j, m) \in \mathbb{N}, 1 \leq j \leq g(m)$. Denote by $p_{m}$ the $m$ th prime number, and let $a_{m}^{(j)} \in \mathbb{C}$. Assume that $g(m) \leq C_{1} p_{m}^{\alpha}$ and $\left|a_{m}^{(j)}\right| \leq p_{m}^{\beta}$ with positive constant $C_{1}$ and non-negative constants $\alpha$ and $\beta$. Define the zeta-function $\widetilde{\varphi}(s)$ by the polynomial Euler product

$$
\widetilde{\varphi}(s)=\prod_{m=1}^{\infty} \prod_{j=1}^{g(m)}\left(1-a_{m}^{(j)} p_{m}^{-s f(j, m)}\right)^{-1} .
$$

The function $\widetilde{\varphi}(s)$ converges absolutely in the region $\sigma>\alpha+\beta+1$, and, in this region, it can be written as the Dirichlet series

$$
\widetilde{\varphi}(s)=\sum_{k=1}^{\infty} \frac{\widetilde{c}_{k}}{k^{s+\alpha+\beta}}
$$

with coefficients $\widetilde{c}_{k}$ such that $\widetilde{c}_{k}=O\left(k^{\alpha+\beta+\varepsilon}\right)$ if all prime factors of $k$ are large for every positive $\varepsilon$ (for the comment, see [11]).

For brevity, denote the shifted version of the function $\widetilde{\varphi}(s)$ as

$$
\varphi(s):=\widetilde{\varphi}(s+\alpha+\beta)=\sum_{k=1}^{\infty} \frac{\widetilde{c}_{k}}{k^{s+\alpha+\beta}}=\sum_{k=1}^{\infty} \frac{c_{k}}{k^{s}}
$$

with $c_{k}:=\widetilde{c}_{k} k^{-\alpha-\beta}$. This series is convergent in the half-plane $\sigma>1$.

Suppose that the function $\varphi(s)$ satisfies the following conditions:

(i) $\varphi(s)$ can be continued meromorphically to $\sigma \geq \sigma_{0}$, where $\frac{1}{2} \leq \sigma_{0}<1$, and all poles of $\varphi(s)$ in this region are included in a compact set which has no intersection with the line $\sigma=\sigma_{0}$;

(ii) for $\sigma \geq \sigma_{0}, \varphi(\sigma+i t)=O\left(|t|^{C_{2}}\right)$ with a certain $C_{2}>0$;

(iii) it holds the mean-value estimate

$$
\int_{0}^{T}\left|\varphi\left(\sigma_{0}+i t\right)\right|^{2} d t=O(T) .
$$

We call the set of all such functions $\varphi(s)$ as the class of Matsumoto zetafunctions and denote by $\mathcal{M}$. 
Now, for $\mathbb{N}_{0}:=\mathbb{N} \cup\{0\}$, let $\mathfrak{B}=\left\{b_{m}: m \in \mathbb{N}_{0}\right\}$ be a periodic sequence of complex numbers $b_{m}$ with minimal positive period $l \in \mathbb{N}$. The periodic Hurwitz zeta-function $\zeta(s, \alpha ; \mathfrak{B})$ with parameter $\alpha \in \mathbb{R}, 0<\alpha \leq 1$, is given by the Dirichlet series

$$
\zeta(s, \alpha ; \mathfrak{B})=\sum_{m=0}^{\infty} \frac{b_{m}}{(m+\alpha)^{s}} \quad \text { for } \quad \sigma>1 .
$$

It is known that the function $\zeta(s, \alpha ; \mathfrak{B})$ is analytically continued to the whole complex plane, except for a possible simple pole at the point $s=1$ with residue $b:=\frac{1}{l} \sum_{m=0}^{l-1} b_{m}$. If $b=0$, then $\zeta(s, \alpha ; \mathfrak{B})$ is an entire function.

It is possible to prove functional limit theorems for the whole class $\mathcal{M}$, but it is difficult to prove the denseness lemma which is necessary to prove the universality theorem. Therefore in the proof of the universality we use an assumption that the function $\varphi(s)$ belongs to the Steuding class $\widetilde{S}$ defined below.

We say that the function $\varphi(s)$ belongs to the class $\widetilde{S}$ if the following conditions are satisfied:

(a) there exists a Dirichlet series expansion $\varphi(s)=\sum_{m=1}^{\infty} a(m) m^{-s}$ with $a(m)=O\left(m^{\varepsilon}\right)$ for every $\varepsilon>0$;

(b) there exists $\sigma_{\varphi}<1$ such that $\varphi(s)$ can be meromorphically continued to the half-plane $\sigma>\sigma_{\varphi}$ and is holomorphic except for a pole at $s=1$;

(c) for every fixed $\sigma>\sigma_{\varphi}$ and $\varepsilon>0$, the order estimate $\varphi(\sigma+i t)=O\left(|t|^{C_{3}+\varepsilon}\right)$ with $C_{3} \geq 0$ holds;

(d) there exists the Euler product expansion over primes, i.e.,

$$
\varphi(s)=\prod_{p \in \mathbb{P}} \prod_{j=1}^{l}\left(1-a_{j}(p) p^{-s}\right)^{-1} ;
$$

(e) there exists a constant $\kappa>0$ such that

$$
\lim _{x \rightarrow \infty} \frac{1}{\pi(x)} \sum_{p \leq x}|a(p)|^{2}=\kappa,
$$

where $\pi(x)$ denotes the number of primes $p$ up to $x$.

For $\varphi(s) \in \widetilde{S}$, let $\sigma^{*}$ be the infimum of all $\sigma_{1}$ such that

$$
\frac{1}{2 T} \int_{-T}^{T}|\varphi(\sigma+i t)|^{2} \mathrm{~d} t \sim \sum_{m=1}^{\infty} \frac{|a(m)|^{2}}{m^{2 \sigma}}
$$

holds for any $\sigma \geq \sigma_{1}$. Then $\frac{1}{2} \leq \sigma^{*}<1$, and we see that $\widetilde{S} \subset \mathcal{M}$ if $\sigma_{0}=\sigma^{*}+\varepsilon$ is chosen. Note that the class $\widetilde{S}$ is not a subclass of the Selberg class!

To formulate mixed joint discere limit theorems and universality property for the tuple $(\varphi(s), \zeta(s, \alpha ; \mathfrak{B}))$ and further results, we need some notation. For 
any compact set $K \subset \mathbb{C}$, denote by $H^{c}(K)$ the set of all $\mathbb{C}$-valued functions defined on $K$, continuous on $K$ and holomorphic in the interior of $K$. By $H_{0}^{c}(K)$ denote the subset of $H^{c}(K)$ such that, on $K$, all elements of $H^{c}(K)$ are non-vanishing. Let $D(a, b)=\{s \in \mathbb{C}: a<\sigma<b\}$ for $a, b \in \mathbb{R}, a<b$, and we denote by meas $\{A\}$ the Lebesgue measure of the measurable set $A \subset \mathbb{R}$. For any set $S, \mathcal{B}(S)$ denotes the set of all Borel subsets of $S$, and, for any region $D, H(D)$ denotes the set of all holomorphic functions on $D$.

The first result on the mixed joint universality of the pair $(\varphi(s), \zeta(s, \alpha ; \mathfrak{B}))$ is the following theorem, which considers the situation when the shifting parameter is moving continuously.

Theorem 1 [Theorem 2.2, [9]]. Suppose $\varphi(s) \in \widetilde{S}$, and $\alpha$ is a transcendental number, $0<\alpha<1$. Let $K_{1}$ be a compact subset of the strip $D\left(\sigma^{*}, 1\right), K_{2}$ be a compact subset of the strip $D\left(\frac{1}{2}, 1\right)$, both with connected complements. Suppose that $f_{1}(s) \in H_{0}^{c}\left(K_{1}\right), f_{2}(s) \in H^{c}\left(K_{2}\right)$. Then, for every $\varepsilon>0$, it holds that

$$
\begin{aligned}
\liminf _{T \rightarrow \infty} \frac{1}{T} \operatorname{meas}\{\tau \in[0, T] & \sup _{s \in K_{1}}\left|\varphi(s+i \tau)-f_{1}(s)\right|<\varepsilon, \\
& \left.\sup _{s \in K_{2}}\left|\zeta(s+i \tau, \alpha ; \mathfrak{B})-f_{2}(s)\right|<\varepsilon\right\}>0 .
\end{aligned}
$$

More interesting and complicated questions on mixed joint universality are concerning the discrete case, when shifting parameters take discrete values. The first result of such kind is the discrete analogue of Theorem 1, which was proved by the authors in [10].

Let $h>0$ be the shifting parameter, that is the common difference of the relevant arithmetic progression, and put

$$
L(\alpha, h):=\left\{(\log p: p \in \mathbb{P}),\left(\log (m+\alpha): m \in \mathbb{N}_{0}\right), 2 \pi / h\right\}
$$

We call $L(\alpha, h)$ is linearly independent over $\mathbb{Q}$ when the relation

$$
\sum_{p \in \mathbb{P}} k_{p} \log p+\sum_{m \in \mathbb{N}_{0}} l_{m} \log (m+\alpha)+a 2 \pi / h=0
$$

(where $k_{p}, l_{m}, a \in \mathbb{Z}$ and only a finite number of them are nonzero) holds if and only if all $k_{p}, l_{m}, a$ are zero.

Remark 1. In [12], we wrote the set $L(\alpha, h)$ in a slightly different way:

$$
L(\alpha, h):=\{\log p: p \in \mathbb{P}\} \cup\left\{\log (m+\alpha): m \in \mathbb{N}_{0}\right\} \cup\{2 \pi / h\} .
$$

However this notation may cause a misunderstanding, because if we choose $h=2 \pi / \log \left(m_{0}+\alpha\right)$ with a certain $m_{0} \in \mathbb{N}_{0}$, then $L(\alpha, h)=\{\log p: p \in \mathbb{P}\} \cup$ $\left\{\log (m+\alpha): m \in \mathbb{N}_{0}\right\}$, and even if the elements of this set are linearly independent, the required condition on (1.1) cannot be satisfied. This matter was pointed out by Professor Antanas Laurinčikas, to whom the authors express their sincere thanks. 
Theorem 2 [Theorem 3, [10]]. Suppose $\varphi(s) \in \widetilde{S}$, and that the set $L(\alpha, h)$ is linearly independent over $\mathbb{Q}$. Let $K_{1}, K_{2}, f_{1}(s)$ and $f_{2}(s)$ be the same as in Theorem 1. Then, for every $\varepsilon>0$, it holds that

$$
\begin{aligned}
\liminf _{N \rightarrow \infty} \frac{1}{N+1} \#\{0 \leq k \leq N & \sup _{s \in K_{1}}\left|\varphi(s+i k h)-f_{1}(s)\right|<\varepsilon, \\
& \left.\sup _{s \in K_{2}}\left|\zeta(s+i k h, \alpha ; \mathfrak{B})-f_{2}(s)\right|<\varepsilon\right\}>0 .
\end{aligned}
$$

Here and in what follows, $\#\{A\}$ denotes the cardinality of the set $A$.

In [12], we have extended our investigations and studied the case when common differences of arithmetic progressions for both of the zeta-functions in the tuple are different.

The aim of this paper is to show more general results than those mentioned above. Here we prove two mixed joint universality theorems - in the cases of the same and different common differences of arithmetic progressions. The novelty is that a wide collection of the periodic Hurwitz zeta-functions $\zeta\left(s, \alpha_{j} ; \mathfrak{B}_{j l}\right)$ will be constructed; here for each $\alpha_{j}$ a collection of sequences $\mathfrak{B}_{j l}$ is attached. This type of general collection of periodic Hurwitz zeta-functions has been studied in several previous articles (such as $[4,11,16,22]$ and [7]) in the continuous case. In the discrete case, there are papers for periodic zeta-functions (see $[18,19])$. The results in the present article are discrete analogous of Theorem 4.2 from [11], but in [11] we assume the stronger hypothesis, that is the algebraic independence of the parameters $\alpha_{1}, \ldots, \alpha_{r}$.

Suppose that $l(j)$ is a positive integer, $j=1, \ldots, r$, and $\lambda=l(1)+\cdots+l(r)$. For each $j$ and $l, j=1, \ldots, r, l=1, \ldots, l(j)$, let $\mathfrak{B}_{j l}=\left\{b_{m j l} \in \mathbb{C}: m \in \mathbb{N}_{0}\right\}$ be a periodic sequence of complex numbers $b_{m j l}$ (not all zero) with the minimal period $k_{j l}$. Suppose that $\alpha_{j}$ be a real number such that $\alpha_{j} \in(0,1), j=1, \ldots, r$. Let $\zeta\left(s, \alpha_{j} ; \mathfrak{B}_{j l}\right)$ be the corresponding periodic Hurwitz zeta-function for $j=$ $1, \ldots, r, l=1, \ldots, l(j)$. Moreover, let $k_{j}$ the least common multiple of periods $k_{j 1}, \ldots, k_{j l(j)}$, and

$$
B_{j}:=\left(\begin{array}{cccc}
b_{1 j 1} & b_{1 j 2} & \ldots & b_{1 j l(j)} \\
b_{2 j 1} & b_{2 j 2} & \ldots & b_{2 j l(j)} \\
\ldots & \ldots & \ldots & \ldots \\
b_{k_{j} j 1} & b_{k_{j} j 2} & \ldots & b_{k_{j} j l(j)}
\end{array}\right), \quad j=1, \ldots, r .
$$

Now let $\underline{\alpha}:=\left(\alpha_{1}, \ldots, \alpha_{r}\right), h>0$, and let $\underline{h}:=\left(h_{1}, h_{21}, \ldots, h_{2 r}\right)$ for $h_{1}>0$ and $h_{2 j}>0$ with $j=1, . ., r$. Define two sets

$$
\begin{aligned}
L(\underline{\alpha}, h): & =L\left(p, \alpha_{1}, \ldots, \alpha_{r}, h\right) \\
& =\left\{(\log p: p \in \mathbb{P}),\left(\log \left(m+\alpha_{j}\right): m \in \mathbb{N}_{0}, j=1, \ldots, r\right), 2 \pi / h\right\}, \\
L(\underline{\alpha}, \underline{h}): & =L\left(p, \alpha_{1}, \ldots, \alpha_{r}, h_{1}, h_{21}, \ldots, h_{2 r}\right) \\
& =\left\{\left(h_{1} \log p: p \in \mathbb{P}\right),\left(h_{2 j} \log \left(m+\alpha_{j}\right): m \in \mathbb{N}_{0}, j=1, \ldots, r\right), \pi\right\} .
\end{aligned}
$$

The meaning of the linear independence of these sets is similar to that of $L(\alpha, h)$.

Our first new discrete mixed joint universality theorem is as follows. 
Theorem 3. Suppose that the set $L(\underline{\alpha}, h)$ is linearly independent over $\mathbb{Q}$, $\operatorname{rank} B_{j}=l(j), j=1, \ldots, r$, and $\varphi(s)$ belongs to the class $\widetilde{S}$. Let $K_{1}$ be a compact subset of $D\left(\sigma^{*}, 1\right), K_{2 j l}$ be compact subsets of $D\left(\frac{1}{2}, 1\right), l=1, \ldots, l(j)$, all of them with connected complements. Suppose that $f_{1} \in H_{0}^{c}\left(K_{1}\right)$ and $f_{2 j l} \in H^{c}\left(K_{2 j l}\right)$. Then, for every $\varepsilon>0$, it holds that

$$
\begin{aligned}
\liminf _{N \rightarrow \infty} \frac{1}{N+1} \#\left\{0 \leq k \leq N: \sup _{s \in K_{1}}\left|\varphi(s+i k h)-f_{1}(s)\right|<\varepsilon,\right. \\
\left.\quad \sup _{1 \leq j \leq r} \sup _{1 \leq l \leq l(j)} \sup _{s \in K_{2 j l}}\left|\zeta\left(s+i k h, \alpha_{j} ; \mathfrak{B}_{j l}\right)-f_{2 j l}(s)\right|<\varepsilon\right\}>0 .
\end{aligned}
$$

In Theorem 3, there appears only one common difference $h$. Our second new theorem on universality describes the situation when the common differences associated with relevant zeta-functions can be different from each other.

Theorem 4. Suppose that the set $L(\underline{\alpha}, \underline{h})$ is linearly independent over $\mathbb{Q}$, and $B_{j}, f_{1}(s), f_{2 j l}(s), K_{1}, K_{2 j l}$ and $\varphi(s)$ satisfy hypotheses of Theorem 3. Then, for every $\varepsilon>0$, it holds that

$$
\begin{aligned}
\liminf _{N \rightarrow \infty} \frac{1}{N+1} \#\left\{0 \leq k \leq N: \sup _{s \in K_{1}}\left|\varphi\left(s+i k h_{1}\right)-f_{1}(s)\right|<\varepsilon,\right. \\
\left.\sup _{1 \leq j \leq r} \sup _{1 \leq l \leq l(j)} \sup _{s \in K_{2 j l}}\left|\zeta\left(s+i k h_{2 j}, \alpha_{j} ; \mathfrak{B}_{j l}\right)-f_{2 j l}(s)\right|<\varepsilon\right\}>0 .
\end{aligned}
$$

Remark 2. As we already have noted, Theorems 3 and 4 are discrete analogues of Theorem 4.2 from [11], but here, instead of algebraic independence of the parameters $\alpha_{1}, \ldots, \alpha_{r}$, the linear independence of the elements of the sets $L(\underline{\alpha}, h)$ and $L(\underline{\alpha}, \underline{h})$ over the set of rational numbers $\mathbb{Q}$ are used, respectively.

\section{A discrete limit theorem}

Theorem 3 is obviously a special case of Theorem 4, so it is enough to prove Theorem 4. For this aim, we use a discrete mixed joint limit theorem (Theorem 5 below) in the sense of weakly convergent probability measures in the space of analytic functions. Since Theorem 5 is valid for more general $\varphi(s) \in$ $\mathcal{M}$, we will formulate the theorem in such a general setting. In this section, we first prove two auxiliary lemmas which play essential roles in the proof of Theorem 5 .

We introduce certain topological structure. Let $\gamma$ be the unit circle on the complex plane, and $\Omega_{1}$ and $\Omega_{2}$ be two tori defined as $\Omega_{1}=\prod_{p \in \mathbb{P}} \gamma_{p}, \Omega_{2}=\prod_{m=0}^{\infty} \gamma_{m}$, where $\gamma_{p}=\gamma$ for all $p \in \mathbb{P}$, and $\gamma_{m}=\gamma$ for all $m \in \mathbb{N}_{0}$, respectively. It is well known that both tori $\Omega_{1}$ and $\Omega_{2}$ are compact topological Abelian groups with respect to the product topology and pointwise multiplication. Therefore,

$$
\underline{\Omega}:=\Omega_{1} \times \Omega_{21} \times \cdots \times \Omega_{2 r}
$$

with $\Omega_{2 j}=\Omega_{2}$ for all $j=1, \ldots, r$ also is a compact topological group. This gives that we can define the probability Haar measure $m_{H}$ on $(\underline{\Omega}, \mathcal{B}(\underline{\Omega}))$, where $m_{H}$ 
is the product of Haar measures $m_{H 1}$ and $m_{H 2 j}, j=1, \ldots, r$, defined on spaces $\left(\Omega_{1}, \mathcal{B}\left(\Omega_{1}\right)\right)$ and $\left(\Omega_{2 j}, \mathcal{B}\left(\Omega_{2 j}\right)\right), j=1, \ldots, r$, respectively. This fact allows us to have the probability space $\left(\underline{\Omega}, \mathcal{B}(\underline{\Omega}), m_{H}\right)$ (for the details, see [11]).

Denote by $\omega_{1}(p)$ be the projection of $\omega_{1} \in \Omega_{1}$ to $\gamma_{p}, p \in \mathbb{P}$, and by $\omega_{2 j}(m)$ the projection of $\omega_{2 j} \in \Omega_{2 j}$ to $\gamma_{m}, m \in \mathbb{N}_{0}, j=1, \ldots, r$. The definition of $\omega_{1}(m)$ for general $m \in \mathbb{N}_{0}$ is given by $\omega_{1}(m)=\omega_{1}\left(p_{1}\right)^{a_{1}} \cdots \omega_{1}\left(p_{q}\right)^{a_{q}}$ according to the decomposition $m=p_{1}^{a_{1}} \cdots p_{q}^{a_{q}}$ into prime divisors. Let $\underline{\omega}=\left(\omega_{1}, \omega_{21}, \ldots, \omega_{2 r}\right)$ be an element of $\underline{\Omega}$.

For $A \in \mathcal{B}(\underline{\Omega})$, on $(\underline{\Omega}, \mathcal{B}(\underline{\Omega}))$, define

$$
\begin{aligned}
Q_{N}(A):= & \frac{1}{N+1} \#\left\{0 \leq k \leq N:\left(\left(p^{-i k h_{1}}: p \in \mathbb{P}\right),\right.\right. \\
& \left.\left.\left(\left(m+\alpha_{1}\right)^{-i k h_{21}}: m \in \mathbb{N}_{0}\right), \ldots,\left(\left(m+\alpha_{r}\right)^{-i k h_{2 r}}: m \in \mathbb{N}_{0}\right)\right) \in A\right\} .
\end{aligned}
$$

Lemma 1. Suppose that the set $L(\underline{\alpha}, \underline{h})$ is linearly independent over $\mathbb{Q}$. Then $Q_{N}$ converges weakly to the Haar measure $m_{H}$ as $N \rightarrow \infty$.

Proof. This is Lemma 6 from [12].

Another important auxiliary result is related with the ergodic theory. It will be used for the identification of the explicit form of the limit measure.

Let $a_{\underline{\alpha}, \underline{h}}:=\left\{\left(p^{-i h_{1}}: p \in \mathbb{P}\right),\left(\left(m+\alpha_{1}\right)^{-i h_{21}}: m \in \mathbb{N}_{0}\right), \ldots,\left(\left(m+\alpha_{r}\right)^{-i h_{2 r}}:\right.\right.$ $\left.\left.m \in \mathbb{N}_{0}\right)\right\}$ be an element of $\underline{\Omega}$. Since the Haar measure $m_{H}$ is an invariant with respect to translations on $\underline{\Omega}$, we define the measurable measure-preserving transformation of the torus $\underline{\Omega}$ by the formula

$$
\Phi_{\underline{\alpha}, \underline{h}}(\underline{\omega}):=a_{\underline{\alpha}, \underline{h}} \underline{\omega}, \quad \underline{\omega} \in \underline{\Omega} .
$$

Recall that the set $A \in \mathcal{B}(\underline{\Omega})$ is invariant with respect to the transformation $\Phi_{\underline{\alpha}, \underline{h}}$ if the sets $A$ and $\Phi_{\underline{\alpha}, \underline{h}}(A)$ differ from each other at most by the set of zero $m_{H}$-measure, and the transformation $\Phi_{\underline{\alpha}, \underline{h}}$ is ergodic if its Borel $\sigma$-field of invariant sets consists of sets having $m_{H}$-measure 0 or 1 .

Lemma 2. Suppose that the set $L(\underline{\alpha}, \underline{h})$ is linearly independent over $\mathbb{Q}$. Then the transformation $\Phi_{\underline{\alpha}, \underline{h}}$ is ergodic.

Proof. This lemma is proved by the Fourier transform method. Any character $\chi$ of the torus $\underline{\Omega}$ can be written in the form

$$
\chi(\underline{\omega})=\prod_{p \in \mathbb{P}} \omega_{1}^{k_{p}}(p) \prod_{m \in \mathbb{N}_{0}} \prod_{j=1}^{r} \omega_{2 j}^{l_{m j}}(m)
$$

where $k_{p}, l_{m 1}, \ldots, l_{m r}$ are integers, only a finite number of which are distinct from 0 . Then

$$
\chi\left(a_{\underline{\alpha}, \underline{h}}\right)=\exp \left(-i h_{1} \sum_{p \in \mathbb{P}} k_{p} \log p-i \sum_{m \in \mathbb{N}_{0}} \sum_{j=1}^{r} h_{2 j} l_{m j} \log \left(m+\alpha_{j}\right)\right) \neq 1
$$


for any non-trivial $\chi$, because of the linear independence of $L(\underline{\alpha}, \underline{h})$. Using this fact, we proceed along the standard way to prove the lemma; see, for example, Section 4 of [2].

Now we are ready for the discrete mixed joint limit theorem for the tuple of the class of zeta-functions under our investigation.

Let $D_{1}$ be an open region of $D\left(\sigma^{*}, 1\right)$, and $D_{2}$ be an open region of $D\left(\frac{1}{2}, 1\right)$. Denote the Cartesian product of $\lambda+1$ such spaces as

$$
\underline{H}=H\left(D_{1}\right) \times \underbrace{H\left(D_{2}\right) \times \ldots \times H\left(D_{2}\right)}_{\lambda} .
$$

Let $\varphi(s) \in \mathcal{M}$, and define

$$
\begin{array}{r}
\underline{Z}(\underline{s}, \underline{\alpha} ; \underline{\mathfrak{B}})=\left(\varphi\left(s_{1}\right), \zeta\left(s_{211}, \alpha_{1} ; \mathfrak{B}_{11}\right), \ldots, \zeta\left(s_{21 l(1)}, \alpha_{1} ; \mathfrak{B}_{1 l(1)}\right), \ldots,\right. \\
\left.\zeta\left(s_{2 r 1}, \alpha_{r} ; \mathfrak{B}_{r 1}\right), \ldots, \zeta\left(s_{2 r l(r)}, \alpha_{r} ; \mathfrak{B}_{r l(r)}\right)\right)
\end{array}
$$

with $\underline{\mathfrak{B}}=\left(\mathfrak{B}_{11}, \ldots, \mathfrak{B}_{1 l(1)}, \ldots, \mathfrak{B}_{r 1}, \ldots, \mathfrak{B}_{r l(r)}\right)$ and $\underline{s}=\left(s_{1}, s_{211}, \ldots, s_{21 l(1)}\right.$, $\left.\ldots, s_{2 r 1}, \ldots, s_{2 r l(r)}\right) \in \mathbb{C}^{\lambda+1}$. Further we write

$$
\begin{aligned}
\underline{s}+i k \underline{h}= & \left(s_{1}+i k h_{1}, s_{211}+i k h_{21}, \ldots, s_{21 l(1)}+i k h_{21}, \ldots,\right. \\
& \left.s_{2 r 1}+i k h_{2 r}, \ldots, s_{2 r l(r)}+i k h_{2 r}\right)
\end{aligned}
$$

for $k \in \mathbb{N}_{0}$. The main tool for the proof of our discrete mixed universality theorems is the limit theorem on $(\underline{H}, \mathcal{B}(\underline{H}))$ for

$$
P_{N}(A):=\frac{1}{N+1} \#\{0 \leq k \leq N: \underline{Z}(\underline{s}+i k \underline{h}, \underline{\alpha} ; \underline{\mathfrak{B}}) \in A\}, \quad A \in \mathcal{B}(\underline{H}) .
$$

On $\left(\underline{\Omega}, \mathcal{B}(\underline{\Omega}), m_{H}\right)$, define the $\underline{H}$-valued random element $\underline{Z}(\underline{s}, \underline{\omega}, \underline{\alpha} ; \underline{\mathfrak{B}})$ by the formula

$$
\begin{array}{r}
\underline{Z}(\underline{s}, \underline{\omega}, \underline{\alpha} ; \underline{\mathfrak{B}})=\left(\varphi\left(s_{1}, \omega_{1}\right), \zeta\left(s_{211}, \alpha_{1}, \omega_{21} ; \mathfrak{B}_{11}\right), \ldots, \zeta\left(s_{21 l(1)}, \alpha_{1}, \omega_{21} ; \mathfrak{B}_{1 l(1)}\right), \ldots,\right. \\
\left.\zeta\left(s_{2 r 1}, \alpha_{r}, \omega_{2 r} ; \mathfrak{B}_{r 1}\right), \ldots, \zeta\left(s_{2 r l(r)}, \alpha_{r}, \omega_{2 r} ; \mathfrak{B}_{r l(r)}\right)\right)
\end{array}
$$

with

$$
\begin{aligned}
& \varphi\left(s_{1}, \omega_{1}\right)=\sum_{m=1}^{\infty} \frac{c_{m} \omega_{1}(m)}{m^{s_{1}}}, \quad s_{1} \in D_{1}, \\
& \zeta\left(s_{2 j l}, \alpha_{j}, \omega_{2 j} ; \mathfrak{B}_{j l}\right)=\sum_{m=0}^{\infty} \frac{b_{m j l} \omega_{2 j}(m)}{\left(m+\alpha_{j}\right)^{s_{2 j l}}}, \quad s_{2 j l} \in D_{2},
\end{aligned}
$$

$j=1, \ldots, r, l=1, \ldots, l(j)$. These series, for almost all $\omega_{1} \in \Omega_{1}$ and $\omega_{2 j} \in \Omega_{2 j}$, $j=1, \ldots, r$, are uniformly convergent on compact subsets of $D_{1}$ and $D_{2}$, respectively (see [6] and [5], respectively). Let $P_{\underline{Z}}$ be the distribution of the random element $\underline{Z}(\underline{s}, \underline{\omega}, \underline{\alpha} ; \underline{\mathfrak{B}})$, i.e., the probability measure on $(\underline{H}, \mathcal{B}(\underline{H}))$ defined as

$$
P_{\underline{Z}}(A)=m_{H}(\underline{\omega} \in \underline{\Omega}: \underline{Z}(\underline{s}, \underline{\omega}, \underline{\alpha} ; \underline{\mathfrak{B}}) \in A), \quad A \in \mathcal{B}(\underline{H}) .
$$


Theorem 5. Suppose that $\varphi(s) \in \mathcal{M}$, and that the set $L(\underline{\alpha}, \underline{h})$ is linearly independent over $\mathbb{Q}$. Then $P_{N}$ converges weakly to $P_{\underline{Z}}$ as $N \rightarrow \infty$.

We prove this theorem in a way whose principle has been already welldeveloped (see, for example, Theorem 4 of [12] or Lemma 5.1 of [11] as the continuous analogue). However our present situation includes a lot of parameters which may cause a trouble for the readers, so we will explain many details even if they are now standard.

\section{Proof of the discrete limit theorem}

We begin with a discrete mixed joint limit theorem for absolutely convergent Dirichlet series. For a fixed number $\sigma_{0}^{*}>\frac{1}{2}$, let

$$
\begin{aligned}
& v_{1}(m, n):=\exp \left(-\left(\frac{m}{n}\right)^{\sigma_{0}^{*}}\right), \quad m \in \mathbb{N}, \quad n \in \mathbb{N}, \\
& v_{2}\left(m, n, \alpha_{j}\right)=\exp \left(-\left(\frac{m+\alpha_{j}}{n+\alpha_{j}}\right)^{\sigma_{0}^{*}}\right), \quad m \in \mathbb{N}_{0}, \quad n \in \mathbb{N}, \quad j=1, \ldots, r .
\end{aligned}
$$

In view of the Mellin transform formula and contour integration, we can show (cf. pp. 153-154 of [13], p. 87 of [20]) that the series

$$
\varphi_{n}\left(s_{1}\right)=\sum_{m=1}^{\infty} \frac{c_{m} v_{1}(m, n)}{m^{s_{1}}}, \quad \zeta_{n}\left(s_{2 j l}, \alpha_{j} ; \mathfrak{B}_{j l}\right)=\sum_{m=0}^{\infty} \frac{b_{m j l} v_{2}\left(m, n, \alpha_{j}\right)}{\left(m+\alpha_{j}\right)^{s_{2 j l}}},
$$

$j=1, \ldots, r, l=1, \ldots, l(r)$, converge absolutely when the real parts of all complex variables are greater than $\frac{1}{2}$. Moreover, for $\underline{\omega} \in \underline{\Omega}$, let

$$
\begin{aligned}
& \varphi_{n}\left(s_{1}, \omega_{1}\right)=\sum_{m=1}^{\infty} \frac{c_{m} \omega_{1}(m) v_{1}(m, n)}{m^{s_{1}}}, \\
& \zeta_{n}\left(s_{2 j l}, \alpha_{j}, \omega_{2 j} ; \mathfrak{B}_{j l}\right)=\sum_{m=0}^{\infty} \frac{b_{m j l} \omega_{2 j}(m) v_{2}\left(m, n, \alpha_{j}\right)}{\left(m+\alpha_{j}\right)^{s_{2 j l}}},
\end{aligned}
$$

$j=1, \ldots, r, l=1, \ldots, l(r)$. These series are also absolutely convergent when the real parts of all complex variables are greater than $\frac{1}{2}$.

Now let us fix $\underline{\widehat{\omega}}=\left(\widehat{\omega}_{1}, \widehat{\omega}_{21}, \ldots, \widehat{\omega}_{2 r}\right) \in \underline{\Omega}$. For $A \in \mathcal{B}(\underline{H})$, define two measures

$$
\begin{aligned}
& P_{N, n}(A):=\frac{1}{N+1} \#\left\{0 \leq k \leq N: \underline{Z}_{n}(\underline{s}+i k \underline{h}, \underline{\alpha} ; \underline{\mathfrak{B}}) \in A\right\}, \\
& P_{N, n, \underline{\widehat{\omega}}}(A):=\frac{1}{N+1} \#\left\{0 \leq k \leq N: \underline{Z}_{n}(\underline{s}+i k \underline{h}, \underline{\widehat{\omega}}, \underline{\alpha} ; \underline{\mathfrak{B}}) \in A\right\},
\end{aligned}
$$

where

$$
\begin{array}{r}
\underline{Z}_{n}(\underline{s}, \underline{\alpha} ; \underline{\mathfrak{B}})=\left(\varphi_{n}\left(s_{1}\right), \zeta_{n}\left(s_{211}, \alpha_{1} ; \mathfrak{B}_{11}\right), \ldots, \zeta_{n}\left(s_{21 l(1)}, \alpha_{1} ; \mathfrak{B}_{1 l(1)}\right), \ldots,\right. \\
\left.\zeta_{n}\left(s_{2 r 1}, \alpha_{r} ; \mathfrak{B}_{r 1}\right), \ldots, \zeta_{n}\left(s_{2 r l(r)}, \alpha_{r} ; \mathfrak{B}_{r l(r)}\right)\right)
\end{array}
$$


and

$$
\begin{aligned}
\underline{Z}_{n}(\underline{s}, \underline{\widehat{\omega}}, \underline{\alpha} ; \underline{\mathfrak{B}})= & \left(\varphi_{n}\left(s_{1}, \widehat{\omega}_{1}\right), \zeta_{n}\left(s_{211}, \alpha_{1}, \widehat{\omega}_{21} ; \mathfrak{B}_{11}\right), \ldots, \zeta_{n}\left(s_{21 l(1)}, \alpha_{1}, \widehat{\omega}_{21} ; \mathfrak{B}_{1 l(1)}\right),\right. \\
& \left.\ldots, \zeta_{n}\left(s_{2 r 1}, \alpha_{r}, \widehat{\omega}_{2 r} ; \mathfrak{B}_{r 1}\right), \ldots, \zeta_{n}\left(s_{2 r l(r)}, \alpha_{r}, \widehat{\omega}_{2 r} ; \mathfrak{B}_{r l(r)}\right)\right) .
\end{aligned}
$$

Lemma 3. Suppose that the set $L(\underline{\alpha}, \underline{h})$ is linearly independent over $\mathbb{Q}$. Then, for all $n$, both the measures $P_{N, n}$ and $P_{N, n, \widehat{\omega}}$ converge weakly to the same probability measure (denote it by $\left.P_{n}\right)$ on $(\underline{H}, \mathcal{B}(\underline{H})$ ) as $N \rightarrow \infty$.

Proof. This is a generalization of Lemma 1 of [12]. The following proof is similar to the argument included in several previous articles, such as $[4,17]$. Define $h_{n}: \underline{\Omega} \rightarrow \underline{H}$ by $h_{n}(\underline{\omega})=\underline{Z}_{n}(\underline{s}, \underline{\omega}, \underline{\alpha} ; \underline{\mathfrak{B}})$. This is continuous, and

$$
\begin{aligned}
& h_{n}\left(\left(p^{-i k h_{1}}: p \in \mathbb{P}\right),\left(\left(m+\alpha_{1}\right)^{-i k h_{21}}: m \in \mathbb{N}_{0}\right), \ldots,\left(\left(m+\alpha_{r}\right)^{-i k h_{2 r}}: m \in \mathbb{N}_{0}\right)\right) \\
& =\underline{Z}_{n}(\underline{s}+i k \underline{h}, \underline{\alpha} ; \underline{\mathfrak{B}}) .
\end{aligned}
$$

Therefore, $P_{N, n}=Q_{N} \circ h_{n}^{-1}$. This fact, Lemma 1 and Theorem 5.1 of [1] give that $P_{N, n}$ converges weakly to $m_{H} \circ h_{n}^{-1}$ as $N \rightarrow \infty$.

Next define $g_{n}: \underline{\Omega} \rightarrow \underline{H}$ by $g_{n}(\underline{\omega})=h_{n}(\underline{\omega} \cdot \underline{\widehat{\omega}})$. Then

$$
\begin{aligned}
& g_{n}\left(\left(p^{-i k h_{1}}: p \in \mathbb{P}\right),\left(\left(m+\alpha_{1}\right)^{-i k h_{21}}: m \in \mathbb{N}_{0}\right), \ldots,\left(\left(m+\alpha_{r}\right)^{-i k h_{2 r}}: m \in \mathbb{N}_{0}\right)\right) \\
& =\underline{Z}_{n}(\underline{s}+i k \underline{\underline{h}}, \underline{\widehat{\omega}}, \underline{\alpha} ; \underline{\mathfrak{B}}),
\end{aligned}
$$

so $P_{N, n, \widehat{\omega}}=Q_{N} \circ g_{n}^{-1}$ which converges weakly to $m_{H} \circ g_{n}^{-1}$ as above. Therefore, the lemma follows, because the invariance property of the Haar measure implies that $m_{H} \circ g_{n}^{-1}=m_{H} \circ h_{n}^{-1}$.

In the next step of the proof, we pass from $\underline{Z}_{n}(\underline{s}, \underline{\alpha} ; \underline{\mathfrak{B}})$ and $\underline{Z}_{n}(\underline{s}, \underline{\omega}, \underline{\alpha} ; \underline{\mathfrak{B}})$ to $\underline{Z}(\underline{s}, \underline{\alpha} ; \underline{\mathfrak{B}})$ and $\underline{Z}(\underline{s}, \underline{\omega}, \underline{\alpha} ; \underline{\mathfrak{B}})$ by the approximation in mean, respectively. First define a metric $\varrho$ on $\underline{H}$. For any open region $G \subset \mathbb{C}$, let $\rho_{G}$ be the standard metric on $H(G)$ which induces the topology of uniform convergence on compact subsets (see Section 1.7 of [14]). Then, for two elements

$$
\begin{aligned}
& \underline{f}=\left(f_{1}, f_{211}, \ldots, f_{21 l(1)}, \ldots, f_{2 r 1}, \ldots, f_{2 r l(r)}\right), \\
& \underline{g}=\left(g_{1}, g_{211}, \ldots, g_{21 l(1)}, \ldots, g_{2 r 1}, \ldots, g_{2 r l(r)}\right)
\end{aligned}
$$

of $\underline{H}$, define the metric $\underline{\varrho}$ by

$$
\underline{\varrho}(\underline{f}, \underline{g})=\max \left\{\rho_{D_{1}}\left(f_{1}, g_{1}\right), \max _{1 \leq j \leq r} \max _{1 \leq l \leq l(j)} \rho_{D_{2}}\left(f_{2 j l}, g_{2 j l}\right)\right\} .
$$

Lemma 4. Let $L(\underline{\alpha}, \underline{h})$ be as in Theorem 5. Then we have

$$
\lim _{n \rightarrow \infty} \limsup _{N \rightarrow \infty} \frac{1}{N+1} \sum_{k=0}^{N} \underline{\varrho}\left(\underline{Z}(\underline{s}+i k \underline{h}, \underline{\alpha} ; \underline{\mathfrak{B}}), \underline{Z}_{n}(\underline{s}+i k \underline{h}, \underline{\alpha} ; \underline{\mathfrak{B}})\right)=0
$$

and, for almost all $\underline{\omega} \in \underline{\Omega}$,

$$
\lim _{n \rightarrow \infty} \limsup _{N \rightarrow \infty} \frac{1}{N+1} \sum_{k=0}^{N} \underline{\varrho}\left(\underline{Z}(\underline{s}+i k \underline{h}, \underline{\omega}, \underline{\alpha} ; \underline{\mathfrak{B}}), \underline{Z}_{n}(\underline{s}+i k \underline{h}, \underline{\omega}, \underline{\alpha} ; \underline{\mathfrak{B}})\right)=0 .
$$


Proof. This is a generalization of Lemma 2 of [12]. In view of the definition of the metric $\underline{\varrho}$, it is sufficient to show

$$
\lim _{n \rightarrow \infty} \limsup _{N \rightarrow \infty} \frac{1}{N+1} \sum_{k=0}^{N} \rho_{D_{1}}\left(\varphi\left(s_{1}+i k h_{1}\right), \varphi_{n}\left(s_{1}+i k h_{1}\right)\right)=0
$$

and, for almost all $\omega_{1}$,

$$
\lim _{n \rightarrow \infty} \limsup _{N \rightarrow \infty} \frac{1}{N+1} \sum_{k=0}^{N} \rho_{D_{1}}\left(\varphi\left(s_{1}+i k h_{1}, \omega_{1}\right), \varphi_{n}\left(s_{1}+i k h_{1}, \omega_{1}\right)\right)=0
$$

and the corresponding results for periodic Hurwitz zeta-functions.

The proof of (3.1) and (3.2) are included in the proof of Lemma 3 of [10], though the formulas themselves are not explicitly stated there. The corresponding results for periodic Hurwitz zeta-functions are Theorems 4.1 and 4.4 of $[21]$.

In the third step, we introduce one more probability measure, for almost all $\underline{\omega} \in \underline{\Omega}$, defined by

$$
P_{N, \underline{\omega}}(A):=\frac{1}{N+1} \#\{0 \leq k \leq N: \underline{Z}(\underline{s}, \underline{\omega}, \underline{\alpha} ; \underline{\mathfrak{B}}) \in A\}, \quad A \in \mathcal{B}(\underline{H}) .
$$

Lemma 5. Suppose that $L(\underline{\alpha}, \underline{h})$ is as in Theorem 5. Then the measures $P_{N}$ and $P_{N, \underline{\omega}}$ both converge weakly to the same probability measure (denote it by $P)$ on $(\underline{\underline{H}}, \mathcal{B}(\underline{H}))$ as $N \rightarrow \infty$.

Proof. This is a generalization of Lemma 3 of $[12]$. Let $(\widetilde{\Omega}, \mathcal{B}(\widetilde{\Omega}), \widetilde{P})$ be a certain probability space, $\theta_{N}: \widetilde{\Omega} \rightarrow \mathbb{R}$ be a discrete random variable satisfying $\widetilde{P}\left(\theta_{N}=k\right)=\frac{1}{N+1}, k=0, \ldots, N$, and define the $\underline{H}$-valued random element $\underline{X}_{N, n}(\underline{s})$ on $\widetilde{\Omega}$ by

$$
\underline{X}_{N, n}(\underline{s})=\underline{Z}_{n}\left(\underline{s}+i \theta_{N} \underline{h}, \underline{\alpha} ; \underline{\mathfrak{B}}\right) .
$$

Then the distribution of $\underline{X}_{N, n}(\underline{s})$ is clearly $P_{N, n}$.

Let $\underline{X}_{n}(\underline{s})$ be an $\underline{H}$-valued random element whose distribution is $P_{n}$. Then Lemma 3 implies that $\underline{X}_{N, n}(\underline{s})$ converges to $\underline{X}_{n}(\underline{s})$ in distribution as $N \rightarrow \infty$.

We can show that the family $\left\{P_{n}\right\}$ is tight in a standard way (see, for example, pp. 269-270 of [8]). Therefore, by Prokhorov's theorem (see [1]), we can choose a subsequence $\left\{P_{n_{k}}\right\} \subset\left\{P_{n}\right\}$, which converges weakly to a certain probability measure $P$ on $(\underline{H}, \mathcal{B}(\underline{H}))$ as $k \rightarrow \infty$. That is, $\underline{X}_{n_{k}}$ converges to $P$ in distribution as $k \rightarrow \infty$.

Define another random element $\underline{X}_{N}(\underline{s})=\underline{Z}\left(\underline{s}+i \theta_{N} \underline{h}, \underline{\alpha} ; \underline{\mathfrak{B}}\right)$. Then, by Lemma 4, for any $\varepsilon>0$,

$$
\begin{aligned}
\lim _{n \rightarrow \infty} \limsup _{N \rightarrow \infty} \widetilde{P}\left(\underline{\varrho}\left(\underline{X}_{N}(\underline{s}), \underline{X}_{N, n}(\underline{s})\right) \geq \varepsilon\right)=\lim _{n \rightarrow \infty} \limsup _{N \rightarrow \infty} \frac{1}{N+1} \#\{0 \leq k \leq N: \\
\left.\quad \underline{\varrho}\left(\underline{Z}\left(\underline{s}+i \theta_{N} \underline{h}, \underline{\alpha} ; \underline{\mathfrak{B}}\right), \underline{Z}_{n}\left(\underline{s}+i \theta_{N} \underline{h}, \underline{\alpha} ; \underline{\mathfrak{B}}\right)\right) \geq \varepsilon\right\} \\
\leq \lim _{n \rightarrow \infty} \limsup _{N \rightarrow \infty} \frac{1}{\varepsilon(N+1)} \sum_{k=0}^{n} \underline{\varrho}\left(\underline{Z}(\underline{s}+i k \underline{h}, \underline{\alpha} ; \underline{\mathfrak{B}}), \underline{Z}_{n}(\underline{s}+i k \underline{h}, \underline{\alpha} ; \underline{\mathfrak{B}})\right)=0 .
\end{aligned}
$$


Therefore, we can apply Theorem 4.2 of [1] to find that $\underline{X}_{N}(\underline{s})$ converges to $P$ in distribution, that is, $P_{N}$ converges weakly to $P$ as $N \rightarrow \infty$. Moreover, this fact implies that $P$ does not depend on the choice of the subsequence $\left\{P_{n_{k}}\right\}$. Therefore, by Theorem 1.1 .9 of [14], we see that $P_{n}$ converges weakly to $P$ as $n \rightarrow \infty$.

Finally we put

$$
\underline{\widehat{X}}_{N, n}(\underline{s})=\underline{Z}_{n}\left(\underline{s}+i \theta_{N} \underline{h}, \underline{\omega}, \underline{\alpha} ; \underline{\mathfrak{B}}\right) \quad \text { and } \quad \underline{\widehat{X}}_{N}(\underline{s})=\underline{Z}\left(\underline{s}+i \theta_{N} \underline{h}, \underline{\omega}, \underline{\alpha} ; \underline{\mathfrak{B}}\right)
$$

and argue as above. It follows that $\underline{\widehat{X}}_{N, n}(\underline{s}) \rightarrow \underline{X}_{n}(\underline{s})$ as $N \rightarrow \infty$ in distribution. We already mentioned above that $\underline{X}_{n}(\underline{s}) \rightarrow P$ in distribution. Also, using Lemma 4, we find

$$
\lim _{n \rightarrow \infty} \limsup _{N \rightarrow \infty} \widetilde{P}\left(\underline{\varrho}\left(\underline{\widehat{X}}_{N}(\underline{s}), \underline{\widehat{X}}_{N, n}(\underline{s})\right) \geq \varepsilon\right)=0 .
$$

Therefore, again using Theorem 4.2 of [1] we obtain that $P_{N, \underline{\omega}}$ converges weakly to $P$ as $N \rightarrow \infty$.

The final step of the proof is to identify the measure $P$ in Lemma 5.

Lemma 6. The probability measure $P$ coincides with the probability measure $P_{\underline{Z}}$.

Proof. This is a generalization of Lemma 4 of [12]. Using Lemma 2 and the classical Birkhoff-Khintchine theorem (see [3]), we just mimic the standard argument (see, for example, the proof of Theorem 4 of [2]).

The proof of Theorem 5 is completed. From Theorem 5 we can immediately deduce simpler discrete mixed joint limit theorem when the set $L(\underline{\alpha}, \underline{h})$ is replaced by $L(\underline{\alpha}, h)$, that is the case $h_{1}=h_{21}=\cdots=h_{2 j}=h$. Here we give the statement of such discrete mixed joint limit theorem.

Theorem 6. Suppose that $\varphi(s) \in \mathcal{M}$, the set $L(\underline{\alpha}, h)$ is linearly independent over $\mathbb{Q}$ and $\operatorname{rank}\left(B_{j}\right)=l(j), j=1, \ldots, r$. Then $P_{N}$ converges weakly to $P_{\underline{Z}}$ as $N \rightarrow \infty$.

\section{Proof of Theorems 3 and 4}

For the proof of universality property in the Voronin sense, we need to construct the support of the probability measure $P_{\underline{Z}}$ in an explicit form. Assume that $\varphi(s) \in \widetilde{S}$, and let $K_{1}, K_{2 j l}, f_{1}(s), f_{2 j l}, j=1, \ldots, r, l=1, \ldots, l(j)$, be as in our most general universality result, i.e., Theorem 4 . Suppose that $M>0$ is a sufficiently large number such that $K_{1}$ belongs to

$$
D_{M}=\left\{s \in \mathbb{C}: \sigma_{0}<\sigma<1,|t|<M\right\} .
$$

Since, by the conditions (i) and (b), the function $\varphi(s)$ has just one pole at $s=1$, define $D_{\varphi}=\left\{s \in \mathbb{C}: \sigma>\sigma_{0}, \sigma \neq 1\right\}$. This gives us that $D_{M} \subset D_{\varphi}$. Moreover 
we can find $T>0$ such that each $K_{2 j l}$, for $j=1, \ldots, r$ and $l=1, \ldots, l(j)$, is a part of

$$
D_{T}=\left\{s \in \mathbb{C}: \frac{1}{2}<\sigma<1,|t|<T\right\} .
$$

Now we choose $D_{1}=D_{M}$ and $D_{2}=D_{T}$ and consider an explicit form of the support $S_{\underline{Z}}$ of the probability measure $P_{\underline{Z}}$. Let $S_{\varphi}:=\left\{f \in H\left(D_{M}\right): f(s) \neq\right.$ 0 for $D_{M}$, or $\left.f(s) \equiv 0\right\}$.

Lemma 7. Suppose that the set $L(\underline{\alpha}, \underline{h})$ is linearly independent over $\mathbb{Q}$. Then the support of the measure $P_{\underline{Z}}$ is the set $S_{\underline{Z}}:=S_{\varphi} \times H^{\lambda}\left(D_{T}\right)$.

Proof. This is Lemma 5.2 of [11]. In [11], no proof is given, so here we present a proof. We want to find the minimal closed set $A$ which satisfies $P_{\underline{Z}}(A)=1$. It is sufficient to consider the set of the form $A=A_{1} \times A_{21} \times \cdots \times \bar{A}_{2 r}$, where $A_{1} \in \mathcal{B}\left(H\left(D_{M}\right)\right)$ and $A_{2 j} \in \mathcal{B}\left(H\left(D_{T}\right)^{l(j)}\right), 1 \leq j \leq r$. Then

$$
\begin{gathered}
P_{\underline{Z}}(A)=m_{H}(\underline{\omega} \in \underline{\Omega}: \underline{Z}(\underline{s}, \underline{\omega}, \underline{\alpha} ; \underline{\mathfrak{B}}) \in A)=m_{1 H}\left(\omega_{1} \in \Omega_{1}: \varphi\left(s_{1}, \omega_{1}\right) \in A_{1}\right) \\
\times \prod_{j=1}^{r} m_{2 H j}\left(\omega_{2 j} \in \Omega_{2 j}:\left(\zeta\left(s_{2 j 1}, \alpha_{j}, \omega_{2 j} ; \mathfrak{B}_{j 1}\right), \ldots,\right.\right. \\
\left.\left.\zeta\left(s_{2 j l(j)}, \alpha_{j}, \omega_{2 j} ; \mathfrak{B}_{j l(j)}\right)\right) \in A_{2 j}\right) .
\end{gathered}
$$

The condition $P_{\underline{Z}}(A)=1$ implies that all factors on the right-hand side are equal to 1 . The minimal closed set $A_{1}$ satisfying $m_{1 H}\left(\omega_{1} \in \Omega_{1}: \varphi\left(s_{1}, \omega_{1}\right) \in\right.$ $\left.A_{1}\right)=1$ is $S_{\varphi}$ (see Lemma 5.12 of [26]). Under the assumption $\operatorname{rank} B_{j}=l(j)$, the minimal closed set $A_{2 j}$ satisfying $m_{2 H j}\left(\omega_{2 j} \in \Omega_{2 j}:\left(\zeta\left(s_{2 j 1}, \alpha_{j}, \omega_{2 j} ; \mathfrak{B}_{j 1}\right)\right.\right.$, $\left.\left.\ldots, \zeta\left(s_{2 j l(j)}, \alpha_{j}, \omega_{2 j} ; \mathfrak{B}_{j l(j)}\right)\right) \in A_{2 j}\right)=1$ is $H\left(D_{T}\right)^{l(j)}$ for $j=1, \ldots, r$ (see [15], also [22]). Therefore the minimal $A$ is equal to $S_{\varphi} \times H\left(D_{T}\right)^{\lambda}$.

Proof of Theorem 4 (and hence of Theorem 3). The proof of Theorem 4 we obtain by combining results of Theorem 5, Lemma 7 and the Mergelyan theorem on the approximation of analytic functions by polynomials (see [23]). We omit the details since the argument is standard and the same as in Section 4 of [10].

Finally, we would like to mention that probably it is possible to generalize our Theorem 4 in one more direction. In this paper, we consider a wide collection of periodic Hurwitz zeta-functions $\zeta\left(s, \alpha_{j} ; \mathfrak{B}_{j l}\right)$, where we attach the collection of sequences $\left\{\mathfrak{B}_{j l}\right\}, 1 \leq l \leq l(j)$, to a parameter $\alpha_{j}$. However the common differences $h_{2 j}$ are the same for all $\mathfrak{B}_{j l}, l=1, \ldots, l(j)$. The new idea is to consider the situation when to each $\mathfrak{B}_{j l}$ the attached common difference $h_{2 j l}$ may be different.

\section{Acknowledgements}

This work is partially supported by VMU Grants of Clusters Research Projects, No. P-G-19-03, and JSPS Grants-in-Aid for Science Research (B), No. $18 \mathrm{H} 01111$. 


\section{References}

[1] P. Billingsley. Convergence of Probability Measures. Willey, New York, 1999.

[2] E. Buivydas and A. Laurinčikas. A generalized joint discrete universality theorem for the Riemann and Hurwitz zeta-functions. Lith. Math. J., 55(2):193-206, 2015. https://doi.org/10.1007/s10986-015-9273-0.

[3] H. Cramér and M.R. Leadbetter. Stationary and Related Stochastic Processes. Wiley, New York, 1967.

[4] J. Genys, R. Macaitienè, S. Račkauskienė and D. Šiaučiūnas. A mixed joint universality theorem for zeta-functions. Math. Modell. Anal., 15(4):431-446, 2010. https://doi.org/10.3846/1392-6292.2010.15.431-446.

[5] A. Javtokas and A. Laurinčikas. On the periodic Hurwitz zeta-function. HardyRamanujan J., 29:18-36, 2006.

[6] R. Kačinskaitè. A discrete limit theorem for the Matsumoto zetafunction on the complex plane. Lith. Math. J., 40(4):364-378, 2000. https://doi.org/10.1023/A:1007613613949.

[7] R. Kačinskaitè and B. Kazlauskaitè. Two results related to the universality of zeta-functions with periodic coeffcients. Results Math., 73(Article No. 95):73-95, 2018. https://doi.org/10.1007/s00025-018-0856-z.

[8] R. Kačinskaitè and A. Laurinčikas. The joint distribution of periodic zeta-functions. Studia Sci. Math. Hungar., 48(2):257-279, 2011. https://doi.org/10.1556/sscmath.48.2011.2.1162.

[9] R. Kačinskaitè and K. Matsumoto. The mixed joint universality for a class of zeta-functions. Math. Nachr., 288(16):1900-1909, 2015. https://doi.org/10.1002/mana.201400366.

[10] R. Kačinskaitè and K. Matsumoto. On mixed joint discrete universality for a class of zeta-functions. In A. Dubickas et al.(Ed.), Proc. of 6th Intern. Conf. Palanga, Anal. Probab. Methods in Number Theory, pp. 51-66, Vilnius, 2017. Vilnius University Publ. House.

[11] R. Kačinskaitè and K. Matsumoto. Remarks on the mixed joint universality for a class of zeta-functions. Bull. Austral. Math. Soc., 98(2):187-198, 2017. https://doi.org/10.1017/S0004972716000733.

[12] R. Kačinskaitè and K. Matsumoto. On mixed joint discrete universality for a class of zeta-functions II. Lith. Math. J., 59(1):54-66, 2019. https://doi.org/10.1007/s10986-019-09432-1.

[13] A. Laurinčikas. Limit theorems for the Matsumoto zeta-function. J. Théor. Nombres Bordeaux, 8:143-158, 1996. https://doi.org/10.5802/jtnb.161.

[14] A. Laurinčikas. Limit Theorems for the Riemann Zeta-Function. Kluwer Academic Publisher, Dordrecht etc., 1996. https://doi.org/10.1007/978-94-017-20915.

[15] A. Laurinčikas. Voronin-type theorem for periodic Hurwitz zeta-functions. $\quad S b . \quad$ Math., 198:231-242, 2007. https://doi.org/10.1070/SM2007v198n02ABEH003835.

[16] A. Laurinčikas. Joint universality of zeta functions with

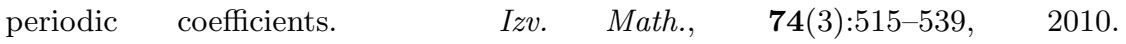
https://doi.org/10.1070/IM2010v074n03ABEH002497. 
[17] A. Laurinčikas. A discrete universality theorem for the Hurwitz zeta-function. J. Number Theory, 143, 2014. https://doi.org/10.1016/j.jnt.2014.04.013.

[18] A. Laurinčikas. Joint discrete universality for periodic zeta-functions. Quaest. Math., 42(5), 2019.

[19] A. Laurinčikas. Joint discrete universality for periodic zeta-functions II. Quaest. Math., 2019. https://doi.org/10.2989/16073606.2019.1654554.

[20] A. Laurinčikas and R. Garunkštis. The Lerch Zeta-Function. Kluwer Academic Publishers, Dordrecht etc., 2002.

[21] A. Laurinčikas and R. Macaitienè. The discrete universality of the periodic Hurwitz zeta-function. Integr. Transf. Spec. Funct., 20:673-686, 2009. https://doi.org/10.1080/10652460902742788.

[22] A. Laurinčikas and S. Skerstonaitè. Joint universality for periodic Hurwitz zeta-functions II. In J. Steuding and R. Steuding(Eds.), Proc. of Conference, Würzburg, Germany, 2008, New Directions in Value-Distribution Theory of Zeta and $L$-Functions, pp. 149-159, Aachen, 2009. Shaker Verlag.

[23] S.N. Mergelyan. Uniform approximations to functions of a complex variable. Am. Math. Soc. Transl., 101:99 p., 1954.

[24] H. Mishou. The joint value-distribution of the Riemann zeta-function and Hurwitz zeta-functions. Lith. Math. J., 47(1):32-47, 2007. https://doi.org/10.1007/s10986-007-0003-0.

[25] J. Sander and J. Steuding. Joint universality for sums and products of Dirichlet L-functions. Analysis (Munich), 26(3):295-312, 2006. https://doi.org/10.1524/anly.2006.26.99.295.

[26] J. Steuding. Value-Distribution of L-Functions, volume 1877 of Lecture Notes Math. Springer Verlag, Berlin etc., 2007.

[27] S.M. Voronin. Theorem on the "universality" of the Riemann zeta-function. Math. USSR Izv., 9:443-453, 1975. https://doi.org/10.1070/IM1975v009n03ABEH001485. 